\title{
Experience of Treating Critically Ill COVID-19 Patients in Daegu, South Korea
}

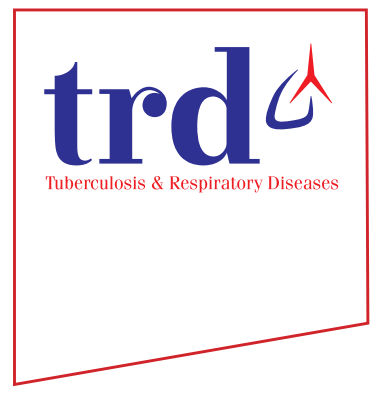

\author{
Kyeong-Cheol Shin, M.D., Ph.D. 10
}

Division of Pulmonology and Allergy, Regional Center for Respiratory Disease, Yeungnam University Medical Center, Yeungnam University College of Medicine, Daegu, Republic of Korea

At the beginning of February 2020, Daegu faced a serious situation due to the rapid outbreak of coronavirus disease 2019 (COVID-19), which is caused by severe acute respiratory syndrome coronavirus-2. The healthcare system in Daegu Metropolitan City was ill-prepared for this sudden disaster situation. The COVID-19 pandemic is still ongoing in South Korea. Daegu has limited medical resources compared to the Seoul Metropolitan Area, and it is hypothesized that a review of the outbreak therein could provide information that will be valuable for dealing with future outbreaks. The likelihood of death due to COVID-19 depends on the capacity of the area for the treatment of critically ill patients. This report reviews the overall treatment process followed for critically ill patients in Daegu.

Keywords: COVID-19; Critical Care; National Intensive Care System; Pandemic

\section{Introduction}

Beginning in February 2020, Daegu faced a serious situation due to the rapid outbreak of coronavirus disease 2019 (COVID-19), which is caused by severe acute respiratory syndrome coronavirus-2 (SARS-CoV-2). The healthcare system in Daegu Metropolitan City was ill-prepared for this sudden disaster situation. The COVID-19 pandemic is still ongoing in South Korea. Daegu has limited medical resources compared to the Seoul Metropolitan Area, and a review of the outbreak therein could provide information valuable for dealing with

Address for correspondence: Kyeong-Cheol Shin, M.D., Ph.D.

Division of Pulmonology and Allergy, Regional Center for Respiratory Disease, Yeungnam University Medical Center, Yeungnam University College of Medicine, 170 Hyeonchung-ro, Nam-gu, Daegu 42415, Republic of Korea

Phone: 82-53-640-6680, Fax: 82-53-654-8386

E-mail: shin1014@ynu.ac.kr

Received: Oct. 12, 2020

Revised: Feb. 2, 2021

Accepted: Mar. 17, 2021

Published online: Mar. 17, 2021

(c) It is identical to the Creative Commons Attribution Non-Commercial License (http://creativecommons.org/licenses/by-nc/4.0/). future outbreaks. The likelihood of death due to COVID-19 depends on the capacity of an area to treat critically ill patients. This report reviews the overall treatment process followed for critically ill patients in Daegu.

\section{Clinical Characteristics of COVID-19 Patients in Daegu}

Most people infected with COVID-19 have mild or no symptoms. However, COVID-19 spread very quickly in Daegu, resulting in many cases in a short period; a large proportion of these were moderate-to-severe and required inpatient treatment with oxygen. In addition, many mild-to-moderate cases progressed to severe disease; these patients tended to deteriorate rapidly, according to the records of a tertiary medical institution in Daegu ${ }^{1}$. Most patients included in this report were over 50 years of age and had severe symptoms and comorbidities. On chest computed tomography, $85 \%$ of patients had multiple lesions or involvement of both lungs. Most critically ill patients progressed to acute respiratory distress syndrome (ARDS) or septic shock, and required mechanical ventilation or extracorporeal membrane oxygenation (ECMO). Among 110 critically ill patients with COVID-19 admitted to the intensive care unit (ICU) in Daegu, septic shock was diagnosed in $22.7 \%(25 / 110)$, and $68.2 \%(75 / 110)$ needed vasopressors. Patients requiring mechanical ventilation had severe hemodynamic instability, and medical staff experienced exhaustion 
because the patients required continuous monitoring. Once intensive care was initiated, it took a long time for the patients to recover, leading to a shortage of beds for other critically ill patients.

\section{Treatment Status of Critically III Patients in Early March 2020}

The number of critically ill COVID-19 patients in Daegu rose between the end of February and middle of March 2020. Table 1 provides data on COVID-19 patients treated in the various medical institutions in Daegu up to March 8, 2020. In total, 1,306 patients were admitted to hospitals in Daegu, among whom 72 received intensive care, 16 were considered critical and transferred to another region, and 32 died. Together, these patients accounted for approximately $9.2 \%$ of all cases. Some of the deceased died in a general ward or emergency room, while others received inadequate treatment. This was because the capacity to treat critically ill patients at medical institutions in Daegu had already been exceeded.

\section{Capacity to Treat Critically III Patients}

\section{Intensive care physicians}

The treatment capacity for critically ill patients depends on the availability of medical facilities and staff. Between 2018 and 2020, five of the 42 tertiary general hospitals in South Korea were in the Daegu-Gyeongbuk region (Kyungpook National University Hospital, Keimyung University Dongsan
Hospital, Daegu Catholic University Medical Center, Yeungnam University Medical Center, and Kyungpook National University Chilgok Hospital). Although Daegu Fatima Hospital is not a tertiary general hospital, it has always provided intensive care. Overall, the medical facilities in Daegu are relatively good compared to other regions of South Korea, but are inferior to those in the Seoul Metropolitan Area.

Intensive care was provided by intensive care and pulmonary specialists (1-2 per hospital). However, some cases received both general and outpatient treatment, and there was a general shortage of intensive care physicians. Table 2 shows the distribution of the physicians responsible for treating critically ill COVID-19 patients among institutions in Daegu. Most of the patients presented with ARDS or septic shock, and there were insufficient numbers of intensive care physicians relative to the volume of cases, as stated above. Although Daegu Dongsan Hospital was initially designated for the treatment of COVID-19 patients with mild disease, an ICU was opened to deal with the dramatic increase in the number of critically ill patients. From March 10, 2020, the operations of the hospital were expanded, facilitated by medical staff from the Korean Society of Critical Care Medicine. Despite this measure, it was evident that more staff were needed on the frontline.

\section{Intensive care facilities}

Negative pressure isolation rooms are needed to treat critically ill COVID-19 patients. All medical institutions in Daegu have 3-4 negative pressure isolation rooms in their ICU; 14 of these satisfy national standards (i.e., are airborne infection isolation rooms) and eight do not. No rooms were available for many of the patients who were already in the ICU, and

Table 1. Data on COVID-19 patients seen at medical institutions in Daegu up to March 8, 2020

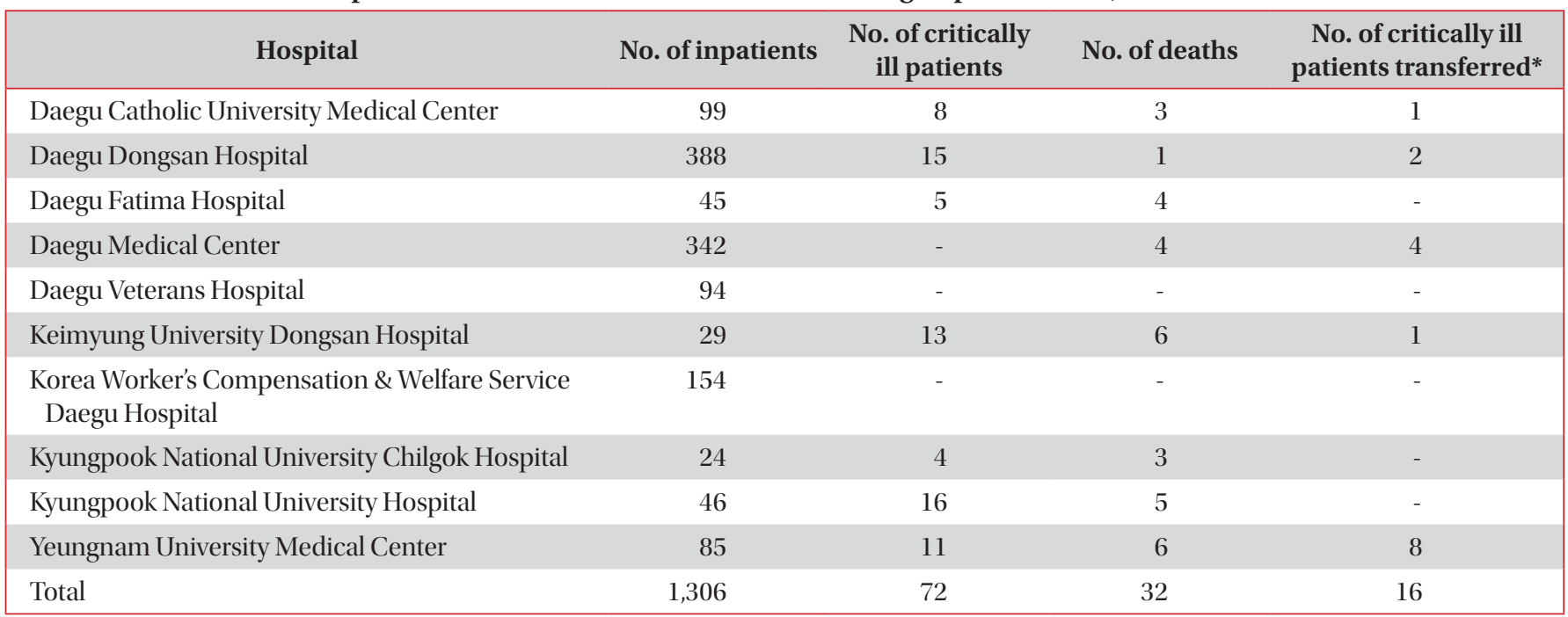

*Critically ill patients transferred to regions outside Daegu.

COVID-19: coronavirus disease 2019. 
Table 2. Distribution of intensive care physicians among medical institutions in Daegu

\begin{tabular}{|c|c|c|c|}
\hline & $\begin{array}{c}\text { Intensive care } \\
\text { specialists }\end{array}$ & $\begin{array}{l}\text { Respiratory medicine } \\
\text { fellows }\end{array}$ & $\begin{array}{l}\text { Internal medicine } \\
\text { residents }\end{array}$ \\
\hline Daegu Catholic University Medical Center & 2 & - & 1 \\
\hline Daegu Dongsan Hospital & 1 & - & - \\
\hline Daegu Fatima Hospital & 2 & - & 1 \\
\hline Keimyung University Dongsan Hospital & 1 & - & 1 \\
\hline Kyungpook National University Chilgok Hospital & 2 & - & - \\
\hline Kyungpook National University Hospital & 2 & - & 1 \\
\hline Yeungnam University Medical Center & 1 & 2 & 1 \\
\hline Total & 11 & 2 & 5 \\
\hline
\end{tabular}

Table 3. Number of beds and negative pressure isolation rooms in ICUs

\begin{tabular}{|c|c|c|c|}
\hline & $\begin{array}{l}\text { Negative pressure isolation } \\
\text { rooms in ICUs }\end{array}$ & $\begin{array}{c}\text { Temporary negative pressure } \\
\text { isolation rooms }\end{array}$ & Total beds \\
\hline Kyungpook National University Hospital & $3($ Feb 19, 2020) & $\begin{array}{l}4(\text { Mar 1, 2020) } \\
5(\text { Mar 6, 2020) }\end{array}$ & 12 \\
\hline Daegu Dongsan Hospital & 3 (Mar 1, 2020) & $\begin{array}{r}4(\text { Feb 29, 2020) } \\
3(\text { Mar 10, 2020) } \\
10^{+}(\operatorname{Mar} 18,2020)\end{array}$ & $10+10$ \\
\hline Keimyung University Dongsan Hospital & $4($ Feb 23, 2020) & & 4 \\
\hline Daegu Catholic University Medical Center & $4^{*}$ & $\begin{array}{l}3(\text { Mar 2, 2020) } \\
6(\text { Mar } 4,2020)\end{array}$ & 9 \\
\hline Daegu Fatima Hospital & $4($ Mar 2, 2020) & $4($ Mar 20, 2020) & 8 \\
\hline Yeungnam University Medical Center & $4($ Feb 26, 2020) & $6(\operatorname{Mar} 6,2020)$ & 10 \\
\hline Kyungpook National University Chilgok Hospital & $2^{*}$ & $5($ Mar 6, 2020) & 5 \\
\hline Total & $18 / 6^{*}$ & $40+10^{\dagger}$ & $58+10$ \\
\hline
\end{tabular}

Parentheses contain the dates on which the intensive care units (ICUs) opened.

*Not operational due to the inability to secure space to transfer existing critically ill patients. 'Ten rooms were operated as "sub-ICUs" (Mar 18, 2020).

the number of beds was insufficient to cope with the large number of critically ill patients who presented at the end of February. Special measures were therefore required to secure additional beds.

\section{Emergency Measures for Treating Critically III Patients in Daegu}

\section{Augmentation of temporary negative pressure isolation rooms in ICUs}

Temporary negative pressure isolation rooms were augmented and, to ensure a sufficient number of beds, patients in the ICU (other than those already in negative pressure isolation rooms) were transferred to another ICU. Three or four portable negative pressure devices were installed to create a large negative pressure region. Although patients with mild disease were supposed to be treated in Daegu Dongsan Hospital, 10 ICU beds were made available to treat the increasing numbers of critically ill patients. Another 10 ICU beds were secured for monitoring patients at risk of rapid deterioration. As shown in Table 3, a total of 58 beds were secured; 40 beds had been added by March 20 (Figure 1). However, by March 8 , while 120 patients needed intensive care, only 51 beds available; thus, the capacity to treat critically ill patients was clearly insufficient.

The reasons for the difficulty in securing negative pressure units for the treatment of critically ill patients were as follows: (1) The exponential increase in the number of patients, (2) Shortage of negative pressure isolation rooms in ICU, (3) Simultaneous treatment of general intensive care patients other than COVID-19, (4) Lack of alternative ICUs to transfer critical patients who were already under intensive care, and (5) De- 


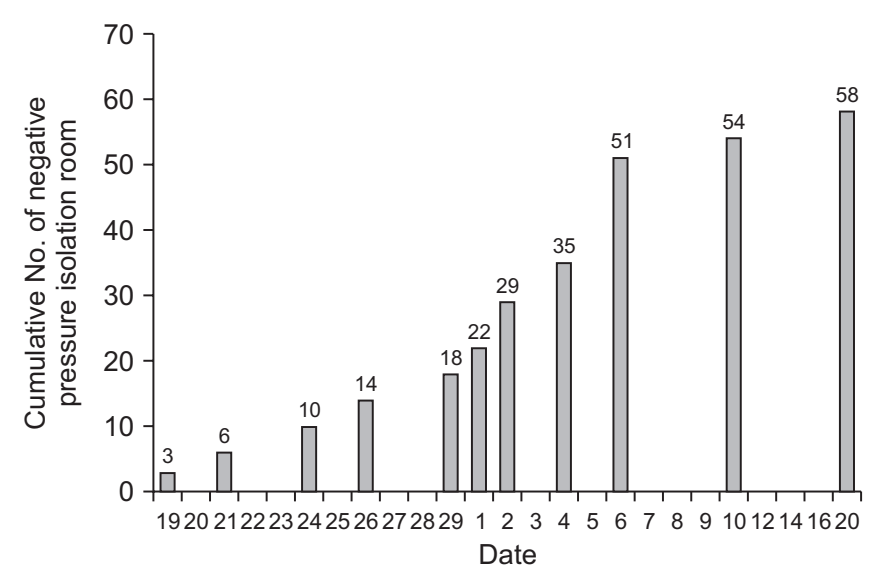

Figure 1. Change in the number of negative pressure isolation rooms in intensive care units from February 19 to March 20, 2020.

layed decision-making on the floor.

With the increase in number of temporary negative pressure isolation rooms, intensive care in Daegu entered a new era. The transfer of critically ill patients to other regions was drastically reduced and very few patients died without first receiving intensive treatment.

The biggest barrier to increasing the number of temporary negative pressure isolation rooms was the potential for crossinfection among patients, who were not isolated on an individual basis. However, this was not discussed with frontline medical staff. The Korea Centers for Disease Control and Prevention guidelines at that time stipulated isolating COVID-19 patients on a one-per-room basis, but this was infeasible given the rapid increase in cases. Ultimately, the responsibility for reducing the risk of cross-infection in the temporary negative pressure isolation rooms fell on the frontline medical staff. To obtain the best treatment outcomes with limited resources, quarantine guidelines must be modified according to the actual situation.

\section{Patient transfer process}

The saturation of intensive care facilities in Daegu necessitated that patients be transferred to other areas. The transportation of severely ill patients was highly complicated. Typically, when transferring a patient at high risk of deterioration, medical staff use the fastest route. However, to transfer a COVID-19 patient, a request first had to be made to the government of Daegu Metropolitan City, and the National Medical Center of Korea then nominated a hospital to treat the patient. The patient could only be transferred with approval from the municipality in which the nominated hospital was located. In some instances, the municipality refused the transfer, while in other cases they did not respond within 24 hours of the transfer request. After several such experiences, it was determined that the requirement for local municipality approval was the most problematic step in the patient transfer process, and we asked the quarantine authorities to resolve this. Eventually, this step was omitted, reducing the difficulty of patient transfer. A systematic and safe transfer system for critically ill COVID-19 patients is urgently required.

\section{Dispatch of intensive care medical staff to Daegu}

Due to the shortage of medical staff to care for critically ill COVID-19 patients in Daegu, the Korean Society of Critical Care Medicine dispatched 32 medical professionals, including 21 intensive care medical specialists, to Daegu Dongsan Hospital between March 10 and April 19, 2020. At that time, severe COVID-19 cases continued to occur in Daegu, but critically ill patients could not be treated because the intensive care facilities had already reached capacity. In addition, even if the number of hospital beds for intensive care had been expanded, there was a lack of medical staff able to provide care for critically ill patients.

Therefore, an intensive care facility was established in Daegu Dongsan Hospital, where patients with mild disease were being treated. Daegu Dongsan Hospital operated as a secondary hospital after Keimyung University Dongsan Hospital had been moved to the Seongseo area of Daegu. It had previously been a tertiary general hospital, and its ICU was still available; this provided 20 beds ( 10 for intensive care, and 10 for monitoring patients at risk of deterioration). The dispatch of intensive care specialists to Daegu represented the first attempt to address the pandemic in South Korea. Specialized medical staff to treat critically ill patients were urgently needed in Daegu, where treatment capacity had already been exceeded and could not be expanded any further to treat new critically ill patients. The staff expressed a desire for enhanced care facilities, rather than transferal of patients to other areas. The number of critically ill patients in Daegu peaked during this period. The intensive care specialists played an important role in this context. The experience of Daegu clearly shows that a detailed initial intensive care plan would have led to better outcomes for patients with severe disease.

\section{Triage}

The severity of each patient's condition prior to hospitalization was determined based on an internally developed triage questionnaire, health insurance data, and the severity of the patient's self-described symptoms at home ${ }^{2}$.

The initial symptoms and underlying health conditions were identified through the triage questionnaire and health insurance data, respectively, as well as via teleconferences attended by a professor from the Daegu Center for Infectious Diseases Control and Prevention and experts from the Daegu Medical Association. The classification process took approximately 2 days. Although a patient classification system was 
proposed by the clinical committee of the National Medical Center of Korea, it was not applicable to patients at home (i.e., prior to hospitalization) because vital signs were required.

Patients with mild symptoms were admitted to Daegu Medical Center and Daegu Dongsan Hospital at the beginning of the outbreak, but as the number of patients increased, extremely mild cases were asked to self-isolate or transfer to a community treatment center, and Daegu Medical Center and Daegu Dongsan Hospital were subsequently assigned mild-to-moderate patients. Severe patients were assigned to a tertiary general hospital (Table 4).

The severity classification schemes differed among the medical institutions; some were based on the National Early Warning Score, but as the number of patients increased rapidly (leading to a shortage of medical staff), severity was sometimes determined based on peripheral oxygen saturation and oxygen administration.

\section{Treatment according to severity}

Treatment may vary depending on the patient volume and management policies. It is very difficult to manage patients based on disease severity if there is a high volume of cases that all require hospitalization. Management based on severity is only feasible when the hospitalization of symptomatic or high-risk patients is prioritized. Furthermore, there must be sufficient medical staff; in particular, the number of medical staff treating patients with mild symptoms should be minimized to reduce the demand on staff treating critically ill patients, and to protect staff from infection. Rapid procurement of sufficient ICU beds is also necessary. Intensive care must be provided as soon as a patient is considered to have severe disease. Therefore, spare beds must be available in the ICU at all times. Due to the shortage of ICU beds in Daegu, critically ill patients were sometimes treated in emergency rooms or general wards.

It is very important to monitor and treat patients at risk of developing ARDS or sepsis appropriately to prevent deterioration. Managing patients likely to become critically ill is actually more difficult than providing intensive care, especially when there are shortages of space and medical staff, as well as a lack of effective treatments. In summary, in Daegu the goal was to treat COVID-19 patients according to disease severity, but the lack of medical resources was a practical limitation.

\section{In-hospital mortality of critically ill COVID-19 patients over 28 days}

The 28-day case fatality rate of 110 patients who received intensive care between February 18 and April 5, 2020 was $42.7 \%^{3}$. This was lower than the rate reported previously in China, of $49 \%{ }^{4}$. The most common SARS-CoV-2 infection-related complication was ARDS (86.4\%), followed by acute cardiac injury, septic shock, and acute kidney injury. The success of ARDS treatment determines the fatality rate among critically ill COVID-19 patients. Some elderly critically ill patients received only conservative, rather than intensive, treatment.

There was no shortage of basic equipment for treating critically ill patients, such as mechanical ventilators. Among 110 critically ill COVID-19 patients admitted to the ICU in Daegu,

Table 4. Assignment of patients to medical institutions according to the severity of symptoms

\begin{tabular}{|c|c|c|c|}
\hline Severity & Criteria & Inpatient medical institution & Medical institution \\
\hline Asymptomatic & No symptoms & $\begin{array}{l}\text { Self-isolation community } \\
\text { treatment center }\end{array}$ & - \\
\hline Mild & Common cold-like symptoms & Public Hospital B & $\begin{array}{l}\text { Armed Forces Daegu Hospital } \\
\text { Daegu Veterans Hospital } \\
\text { Korea Worker's Compensation \& Welfare } \\
\text { Service Daegu Hospital }\end{array}$ \\
\hline $\begin{array}{l}\text { Mild to } \\
\text { moderate }\end{array}$ & Fever $\left(\geq 37.5^{\circ} \mathrm{C}\right)$ and cough & Public Hospital A & $\begin{array}{l}\text { Daegu Dongsan Hospital } \\
\text { Daegu Medical Center }\end{array}$ \\
\hline Severe & $\begin{array}{l}\text { Suspected severe pneumonia } \\
\text { (body temperature } \geq 38.0^{\circ} \mathrm{C} \text { for }>3 \text { days } \\
\text { and respiratory symptoms) }\end{array}$ & Tertiary general hospital & $\begin{array}{l}\text { Daegu Catholic University Medical Center } \\
\text { Yeungnam University Medical Center }\end{array}$ \\
\hline Intensive care & $\begin{array}{l}\text { Suspected critical pneumonia } \\
\text { (dyspnea } \geq 1 \text {-day, respiratory rate } \\
\geq 30 \text { breaths } / \mathrm{min} \text { ) }\end{array}$ & $\begin{array}{l}\text { General hospital, } \\
\text { tertiary general hospital }\end{array}$ & $\begin{array}{l}\text { Daegu Catholic University Medical Center } \\
\text { Daegu Dongsan Hospital } \\
\text { Daegu Fatima Hospital } \\
\text { Keimyung University Dongsan Hospital } \\
\text { Kyungpook National University Chilgok } \\
\text { Hospital } \\
\text { Kyungpook National University Hospital } \\
\text { Yeungnam University Medical Center }\end{array}$ \\
\hline
\end{tabular}


$79(71.8 \%)$ received invasive mechanical ventilation, of whom nine (11.4\%) were placed in the prone position. ECMO treatment was performed in 19 patients in Daegu, 16 of whom were seen at a medical institution providing critical care treatment (such that immediate treatment was possible). Of the 19 patients (17.3\%) who received ECMO treatment, seven were seen at the same hospital. The severity of the patients treated with ECMO may have differed, but considering that $86.4 \%$ of all patients presented with ARDS, it can be assumed that the capability for performing ECMO was different for each hospital. As with severe acute respiratory syndrome and Middle East respiratory syndrome, future viral diseases are also likely to be characterized by severe respiratory complications, such as ARDS. The role of ECMO in the treatment of critically ill patients is being highlighted by the COVID-19 pandemic. Intensive care involving ECMO requires a skilled and experienced team.

\section{Conclusion}

Inevitably, a novel and severe infectious disease will exceed national intensive care resources and cause an increase in deaths. However, excess deaths due to a high concentration of critically ill patients in one area, despite sufficient resources at the national level, suggests a problem with the intensive care system; this was the case in Daegu. Obstacles to intensive care should be eliminated from the national system, and a strategy should be established to meet intensive care demand at the regional level.

\section{Conflicts of Interest}

No potential conflict of interest relevant to this article was reported.

\section{Funding}

No funding to declare.

\section{References}

1. Jang JG, Hur J, Choi EY, Hong KS, Lee W, Ahn JH. Prognostic factors for severe coronavirus disease 2019 in Daegu, Korea. J Korean Med Sci 2020;35:e209.

2. Kim SW, Lee KS, Kim K, Lee JJ, Kim JY; Daegu Medical Association. A brief telephone severity scoring system and therapeutic living centers solved acute hospital-bed shortage during the COVID-19 outbreak in Daegu, Korea. J Korean Med Sci 2020;35:e152.

3. Kim EJ, Lee YH, Park JS, Lee J, Lee SY, Kim Y, et al. Clinical features and prognostic factors of critically ill patients with COVID-19 in Daegu, South Korea: a multi-center retrospective study. Medicine (Baltimore) 2021;100:e24437.

4. Wu Z, McGoogan JM. Characteristics of and important lessons from the coronavirus disease 2019 (COVID-19) outbreak in China: summary of a report of 72314 cases from the Chinese Center for Disease Control and Prevention. JAMA 2020;323:1239-42. 